FERMILAB-Conf-88/81

\title{
Electron and Muon Physics Sessions: Summary*
}

H. E. Montgomery

Fermi National Accelerator Laboratory

P.O. Box 500, Batavia, Dlinois 60510

June 1988

*Summary talk given at the Conference on the Intersections of Nuclear and Particle Physics, Samoset, Maine, May 1988. 
FERMILAB-Conf-88/81

June, 1988

\title{
ELECTRON AND MUON PHYSICS SESSIONS: SUMMARY*
}

\author{
H.E. Montgomery \\ Fermi National Accelerator Laboratory \\ P.O.Box 500, Batavia, I 60510, U.S.A.
}

\begin{abstract}
A short summary is given of the Electron and Muon Physics parallel sessions at the Conference on the Intersections of Nuclear and Particle Physics, Samoset, Maine in May 1988. The sessions occupied approximately 200 minutes, the summary occupied 15 minutes.
\end{abstract}

"Summary talk given at the Conference on the Interweetions of Nuclear and Particle Physics, Samoet, Maine in May 1988. 


\section{INTRODUCTION}

The electromagnetic interaction needs no introduction as a probe of the structure of systems on many scales. The continued use of this technique dominated the sessions on Electron and Muon Physics at the Samoset Meeting. The experimental results continue to stimulate large numbers of theorists and the results on polarized deep inelastic muon scattering and their various interpretations permeated beyond these sessions. The breadth of physics attacked with electrons and muons makes a summary such as this rather peculiar. As one of my nuclear physics friends (I think) commented after my summary, "it was interesting to see Nuclear Physics from a long distance with the telescope inverted". The comment may well be applied to this written version of the summary talk.

\section{WHAT GIVES THE NUCLEON ITS SPIN?}

This year is the 20th anniversary of the presentation[1] of the data from SLAC on the scaling of the inelastic scattering cross-section of electrons from nucleons. One of the most fundamental discoveries which followed in the years that followed was that while all the scattering was from quarks, not all the momentum of the nucleon could be accounted for in the scattering. This of course led to the Gluon being introduced. It is therefore interesting to note that one of the possible conclusions from the recent results on polarized muons scattering from polarized protons is that a small fraction of the spin of the nucleon results from the quarks. The data themselves[2] are a measurement of a polarization asymmetry, which when folded with structure function measurements yield a polarized structure function. There are predictions for the integral, over all $X_{B j}$ of this structure function. It is the construction and interpretation of this integral, which goes beyond the data themselves, which is causing the heat and controversy $[2,3,4]$.

I mentioned above one of the candidate conclusions from these data because it is a favorite observation of mine, that the data on several important structure functions is lacking at low $X_{B j}$ and moderate momentum transfer. This places the evaluation of several quark sum rules[5] on shaky experimental ground. 


\section{STRUCTURE FUNCTIONS, FORM FACTORS; INTER- ACTIONS IMPORTANT}

A full picture of the understanding of structure functions in deep inelastic scattering was presented in the Plenary Sessions. In the parallel session the new results from EMC[7] at CERN and from SLAC-E140[8] now present a rather consistent picture of the ratio between the structure functions of nucleons as measured in moderately heavy nuclei, iron and copper, and those measured on deuterium. One of the components which contributes to the confidence in the result is a determination of the difference between the longitudinal contribution to the scattering from the different targets[8]. The result is consistent with zero and incidentally is a good absolute measurement of the longitudinal to transverse ratio which probably forces a reevaluation of all the structure function data in the SLAC kinematic range. One notes that in all the discussions it is not sufficient to consider the constituents, the partons, but their interactions expressed in terms of QCD corrections or otherwise, are important to describe the data. This is seen also in the new data[9] on the electromagnetic form factor of the deuteron. The electric form factor is comparatively easily described by the models using the basic deuteron constituents; however the magnetic form factor requires[10] a full range of exchange currents.

\section{NUCLEONS IN THE NUCLEUS; $y$ SCALING OR NOT?}

Changing scales, in momentum transfer and energy, drops us into a region where the target is a nucleus and the constituents are nucleons but lots of analogies with high energies are possible. Experimentally, separation of the longitudinal and transverse components of the scattering is normal practice. In general the picture for light nuclei[10,11] is that the quasi elastic scattering data are moderately well described by models and the Coulomb Sum Rule looks reasonable although nucieon correlation effects are evident at low momentum transfer. $y$ scaling is analogous to Bjorken scaling in that the resulting distributions give a representation of the constituent nucleon momentum distribution. There are significant differences[12], of perhaps $40 \%$, between heary and light nuclei and there appears to be inconsistency between longitudinal and transverse responses[13]. However among the heavy nuclei there is little variation. Model calculations[14] in this field suggest that there is no reason 
to expect major manifestations of quark structure as distinct from a conventional description although it is clear that $y$-scaling is broken by the data.

When a final state particle, in addition to the lepton, is observed there are opportunities to further separate the components of the interaction. Such techniques with the wide angle bremsstrahlung as the final state particle now appear to be feasible[15]. The data[16] that exist with a final state proton detected in the direction of the virtual photon have many of the problems with the longitudinal transverse ratio shown by the single arm data. However understanding of the data in terms of the nucleon anomalous magnetic moment can be achieved at the $10 \%$ level with some effort.

\section{REAL PHOTONS; SUPPORT FOR C.I.M.}

Real photons are of course merely a particular subset of virtual photons so they yield very similar types of information. There are now data[17] which compare interactions with an ${ }^{10} \mathrm{O}$ target which yield a final state neutron with those yielding a proton. There are similarities between the data but there is a clear deviation from model expectations at 90 degrees when the photon energy is above about $150 \mathrm{MeV} . \mathrm{A}$ class of models used to describe pion production from nuclei is the $\Delta$-hole model. It appears that rather good agreement can be achieved with the data on $\pi^{0}$ production but charged pion production is by no means as well described[18].

It is interesting to note that some data[19] on deuteron photo-disintegration can be well described by models based on constituent interchange exchange. These models are traditionally associated with high energies and high transverse momenta and these data provide a puzzling twist to the discussion[20] of the validity of extensions of these ideas and QCD to exclusive processes in general.

\section{WEAK INTERACTION EFFECTS}

There have been several important experiments on weak interactions using electron or muon beams, it was gratifying to see that the Parity violation experiment[21] at Bates using polarized electrons scattering from a Carbon target is approaching the level of sensitivity at which it should see the weak-electromagnetic interference. 


\section{CONCLUSIONS}

The interest shown in the electron, muon sessions, the number of contributed papers fully justify the important role which this broad field of physics continues to play. The discussion was lively and open and I for one learned a lot. I acknowledge the efforts of Bill Donnelly who was the joint organizer of this session and who helped me with aspects of the presentation of this summary.

\section{References}

[1] W.K.H. Panofsky, 14th Int. Conf. on High Energy Physics, Vienna 1968, Ed. J. Prentki, J. Steinberger, CERN 1968. E.Bloom et al, Paper \#363 submitted to that Conference.

[2] V. Papavassilliou, talk presented at this conference.

[3] M. Karliner, talk presented at this conference.

[4] F. Close, talk presented at this conference.

[5] See eg. F. Sciulli, Proc. Int. Symp. on Lepton and Photon Interactions at High Energies, Kyoto 1985. Ed. M.Konuma, K.Takahashi, NISEA,Kyoto,1986.

[6] K. Rith, Plenary Talk presented at this conference.

[7] P. Renton, talk presented at this conference.

[8] P. Bosted, talk presented at this conference.

[9] P. Bosted, (second)talk presented at i is conference.

[10] R. Dymarz, talk presented at this conference.

[11] K. Dow, talk presented at this conference.

[12] R. McKeown, talk presented at this conference.

[13] R. Lowry, Plenary Talk presented at this conference.

[14] S. Kumano, talk presented at this conference. 
[15] C. Papanicolas, talk presented at this conference.

[16] G. van der Steenhoven, talk presented at this conference.

[17] E. Beise, talk presented at this conference.

[18] J. Koch, talk presented at this conference.

[19] J. Napolitano, talk presented at this conference.

[20] N. Isgur, Plenary Talk presented at this conference.

[21] P. Souder, talk presented at this conference. 